Asian-Australasian Journal of Food Safety and Security

ISSN 2523-1073 (Print) 2523-2983 (Online)

www.ebupress.com/journal/aajfss

\title{
Article \\ Diarrheal prevalence and risk factors among under five years children in remote coastal area of Bangladesh
}

Md Shafiqul Islam Khan ${ }^{1 *}$, Md. Hasan Al Banna ${ }^{1}$, Sumaiya Akter $^{1}$, Milon Chakma ${ }^{1}$, Shakila ${ }^{1}$, Musammet Rasheda Begum ${ }^{2}$ and Md Nazmul Hassan ${ }^{3}$

${ }^{1}$ Department of Food Microbiology, Patuakhali Science and Technology University, Bangladesh

${ }^{2}$ Department of Agricultural Economics and Social Sciences, Chittagong Veterinary and Animal Sciences University, Khulshi, Chittagong-4225, Bangladesh

${ }^{3}$ Department of Environmental Sanitation, Patuakhali Science and Technology University, Bangladesh

${ }^{*}$ Corresponding author: Md Shafiqul Islam Khan, Department of Food Microbiology, Patuakhali Science and Technology University, Bangladesh. Phone: +8801712115973; E-mail: msikhan312@yahoo.com

Received: 07 November 2018/Accepted: 25 November 2018/ Published: 29 November 2018

\begin{abstract}
Diarrhea causes major childhood morbidity and mortality globally. This study aimed to investigate the prevalence and associated factors of childhood diarrhea among under-five children in Southern coastal area of Bangladesh. A community-based cross-sectional study conducted from April to June 2018 among 202 randomly selected children. A structured questionnaire used to collect information on a three month diseases occurrence and other covariates. The association of diarrhea with socio economy, personal and food hygiene practices observed using multivariate logistic regression after adjusting for potential confounders. Prevalence of childhood diarrhea was $\mathbf{1 0 . 9 \%}$. In bivariate analysis, mother's education, family income, father's occupation, hand washing of child before feeding and refrigerator associated with outcomes. In multivariate analysis, father's occupation, hand washing of child by soap before feeding and having refrigerator in households was associated with childhood diarrhea after controlling the socio-demographic factors (age and gender). Child of unemployed fathers were more likely to have diarrhea than employed father (AOR $=0.206$; 95\% CI: 0.070 0.610). Child do not wash hand before feeding ( $\mathrm{AOR}=0.393 ; 95 \% \mathrm{CI}=0.102-0.511)$ and households not having refrigerator $(\mathrm{AOR}=0.072$; 95\% CI: 0.007-0.745) had higher odds of diarrhea. The load of diarrhea in this setting was almost double to the national rate. Poor hand washing of child and refrigerator are major risk factor high burden of the disease. Health promotion strategies on proper food storage and child feeding practices are recommended for the prevention of childhood diarrhea.
\end{abstract}

Keywords: diarrhea; factor; under five children; Bangladesh

\section{Introduction}

Diarrhea still remained major cause of morbidity and mortality among under-five children in developing countries (Begum et al., 2013; Mahapatra et al., 2015). Majority are aged under five among 1.5 billion cases and 2.5 million death every year for diarrhea (Mannan and Rahman, 2011). Diarrhea accounted for 17\% of all deaths worldwide, 21\% in developing countries and 26.1\% in South Asia (Anteneh et al., 2017; WHO, 2007; Walker et al., 2012). Prevalence of diarrhea among children less than 5 years old was $25.2 \%$ in Kashmir, India and $22 \%$ in Ethiopia (Ahmed et al., 2008; Alebel et al., 2018).

In Bangladesh, 6.1\% under-five children affected and $2.8 \%$ child death by diarrhea (BBS and UNICEF, 2016; Rahman et al., 2014). The lowest prevalence among South Asian countries have made Bangladesh an example for resource poor countries for the advancement in health sectors (Balabanova et al., 2013; Osman, 2008). But morbidity rates is still alarming (Balabanova et al., 2013). In Barisal Division, diarrheal prevalence is 5.0\% and higher prevalence is in city slum areas (BBS and UNICEF, 2016). There is no data regarding the prevalence of 
childhood diarrhea in the coastal poor settings in Bangladesh. Previous study in Kushtia, Bangladesh showed quite alarming rate among children in lower socio economic condition (Khatun et al., 2013). Because economically disadvantaged regions carried the highest burden of under-five (Alebel et al., 2018). There may be a significant gap between the national prevalence and that of this coastal area.

Diarrhea associated with many social, environmental and behavioral aspects of households (Mengistie et al., 2013). Maternal education, availability of latrines and maternal hand washing were the risk factors in Ethiopia (Alebel et al., 2018). Poor kitchen hygiene also proved as a determinant of child diarrhea in Nicaragua (Takanashi, 2009). Lack of access to clean water and sanitation is a major issue in the urban coastal area of Senegal (Thiam et al., 2017).

About 20 million coastal inhabitants have been affected directly or indirectly by flood (Rony et al., 2016). This study conducted in a remote coastal area where flood, river erosion and tidal surges is common (Haque, 2006). Rapid river erosion changes the landscape and excess to clean water in the setting. In addition, risk factors for diarrhea could vary in different settings (Getachew et al., 2018). Identifying modifiable risk factors is a critical step in identifying potential interventions. The findings of this investigation could be useful to policy makers to prevent diarrhea in larger coastal belt or similar setting in Bangladesh.

\section{Materials and Methods}

\subsection{Study setting and design}

We conducted this study among different communities of Dumki sub-district of Patuakhali, Bangladesh, $270 \mathrm{~km}$ away from capital Dhaka. Dumki is river eroded and divided into Lebukhali, Muradia, Pangashia, Sreerampur, Angaria unions (smallest administrative unit). Total population of Dumki was 70705 as of 2001 census data (BBS, 2015). We selected randomly Muradia and Pangashia union to collect the data. We conducted a cross sectional survey to find out the under five children from the household of this area. We conducted a baseline survey to find out the number of target participants. We recruited 202 participants who met our case definition. Households were eligible for inclusion in the survey if the following criteria were met: (i) presence of mothers or caregiver; and (ii) presence of at least one child under the age of five. Children who had diarrhea in last three months considered as case of this study. We registered as case when child passed three or more times loose or watery stools per 24 hours as reported the mother or caregiver (Gidudu et al., 2011). Twelve mothers refused to participate in this study for different causes were excluded. We used purposive sampling method to select the participant. We calculated the sample size from specified proportion test considering 3\% margin of error $95 \%$ CI and 5\% overall prevalence in the Barisal division (BBS and UNICEF, 2016).

\subsection{Procedure}

Ethical clearance was taken and study protocol reviewed by Ethical Committee of Patuakhali Science and Technology University (PSTU), Bangladesh. All caregivers asked for written consent before starting the interview. The anonymity of the respondents is assured before collection of data. Trained investigators collected data by face to face interview using pre-structured questionnaire. The questionnaire consisted of information on socio demography of households, kitchen hygiene, personal hygiene, sanitation and child diarrhea in this area. In this study, we selected positive history of diarrhea as dependent variable. We interviewed mothers or caregivers directly to collect data. We considered only interested mothers or caregivers for this study. We excluded women not interested or absent during data collection from this study. We obtained written consent from the study participants to participate in this study.

\subsection{Statistical analysis}

We used SPSS version 16.0 and SAS version 9.3 to analyze the data. P-values of less than or equal to 0.05 were considered statistically significant. We converted continuous variables into categorical variables to achieve better analysis. Frequency distributions and descriptive statistics for major demographic variables were computed. Bivariate analysis was conducted to assess the relationship between each independent variable and the outcome variable (diarrhea). To control for the effect of confounding factors, multivariate logistic regression was constructed including significant variables in the bivariate analysis. The degree of association between dependent and independent variables were assessed using odds ratio with 95\% confidence interval.

\section{Results}

\subsection{Characteristics of respondents}

Among the households, 190 had 1 child (94.1\%) and 11 had 2 child (6.4\%). Half 101 (50\%) of completed secondary education and $23(11.4 \%)$ had no education. Overall, 191(94.6\%) mothers were housewives. 
Regarding to the education of husband, $61(30.2 \%)$ attended at primary education, and 76(37.6\%) in secondary education and $38(18.8 \%)$ had no education. Employed father were $40(19.8 \%)$. Almost half (48.5\%) of families monthly earned $\leq 10000$ BD Tk.

\subsection{Personal hygiene of mother and child}

All mothers washed their hands before preparation of foods and before feeding children. If child took food themselves, 58(56.3 \%) children washed hand with soap before feeding. When child feed by mother, $1(0.5 \%)$ mothers though ensure child hand washing, 188(99.5\%) not ensure. In case of defecation, 185(91.5\%) mothers washed hands with soap after cleaning child from defecation and 17(8.4\%) did not wash. Almost all mother and child cut baby's nail regularly

\subsection{Sanitary information of households and kitchen}

All households had a private toilet where $131(64.9 \%)$ had Kacha toilet $71(34.7 \%)$ had sanitary (paka) toilet. Toilet located outside for $184(91.1 \%)$ and inside or attached for $18(8.9 \%)$ houses. All families used tube well water for drinking but only 12(5.9\%) families belong to water storage tank. The waste disposal area was open in $200(99.0 \%)$ cases. Among all 202 participants, 192(95\%) used pond water for cleaning utensils and cooking and $10(5.0 \%)$ used tube well water. Only $12(5.9 \%)$ were inside the house and 10(5.0\%) kitchen were with cement floor. For utensil use, 113(55.9\%) did not use separate utensils for keeping raw and cooked foods. Only $39(19.3 \%)$ families had refrigerators and $25(12.5 \%)$ had dustbin for left over.

Table 1. Prevalence and risk factors of child diarrhea.

\begin{tabular}{|c|c|c|c|c|}
\hline \multirow[t]{2}{*}{ Characteristics } & \multicolumn{2}{|c|}{ Diarrhea } & \multicolumn{2}{|c|}{ Odds Ratio (95\% CI) } \\
\hline & Yes $(\%)$ & No $(\%)$ & Unadjusted & Adjusted \\
\hline \multicolumn{5}{|c|}{ Age of child, no. (\%) } \\
\hline $0-2$ Years & $6(3)$ & $48(23.8)$ & $0.963(0.359-2.586)$ & $0.979(0.274-3.495)$ \\
\hline$>2$ years & $17(8.4)$ & $131(64.9)$ & 1 & 1 \\
\hline \multicolumn{5}{|l|}{ Gender, no. (\%) } \\
\hline Male & $13(6.4)$ & $97(48)$ & 1 & $1.808(0.604-5.408)$ \\
\hline Female & $10(5.0)$ & $82(40.6)$ & $0.910(0.379-2.183)$ & 1 \\
\hline \multicolumn{5}{|c|}{ Mother education, no. (\%) } \\
\hline Literate & $13(6.44)$ & $149(73.76)$ & $0.301(0.128-0.829) *$ & $0.327(0.370-3.940)$ \\
\hline Illiterate & $9(4.46)$ & $31(15.35)$ & 1 & 1 \\
\hline \multicolumn{5}{|c|}{ Family size, no. (\%) } \\
\hline Nuclear & $9(4.5)$ & $55(27.2)$ & $1.449(0.592-3.549)$ & \\
\hline Joint & $14(6.9)$ & $124(61.4)$ & 1 & \\
\hline \multicolumn{5}{|c|}{ Family income, no. (\%) } \\
\hline$<=10000$ & $16(7.92)$ & $91(45.05)$ & $2.61(1.024-6.973) *$ & $0.538(0.160-1.806)$ \\
\hline$>10000$ & $6(2.97)$ & $89(44.06)$ & 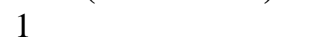 & 1 \\
\hline \multicolumn{5}{|c|}{ Fathers' Occupation, no. (\%); } \\
\hline Unemployed & $14(6.9)$ & $148(73.3)$ & $0.326(0.130-0.820)^{*}$ & $0.206(0.070-0.610) *$ \\
\hline Employed & $9(4.5)$ & $31(15.3)$ & 1 & 1 \\
\hline \multicolumn{5}{|c|}{ Types of toilet, no. (\%); } \\
\hline Kacha & $17(8.4)$ & $115(56.9)$ & $1.577(0.592-4.2)$ & $2.407(0.676-2.570)$ \\
\hline Pacca & $6(3)$ & $64(31.7)$ & 1 & 1 \\
\hline \multicolumn{5}{|c|}{ Hand wash of baby with soap when taking food themselves, no. (\%); $N=103$} \\
\hline Yes & $3(2.91)$ & $55(53.40)$ & $0.015(0.004-0.061) *$ & $0.393(0.102-0.511) *$ \\
\hline No & $35(33.98)$ & $10(9.71)$ & 1 & 1 \\
\hline \multicolumn{5}{|c|}{ Triming of Child nail, no. (\%) } \\
\hline Yes & $22(10.9)$ & $176(87.1)$ & $0.375(0.037-3.763)$ & \\
\hline No & $1(0.5)$ & $3(1.5)$ & 1 & \\
\hline \multicolumn{5}{|c|}{ Use of same utensil, no. (\%) } \\
\hline Yes & $10(5)$ & $79(39.1)$ & $0.974(0.406-2.337)$ & \\
\hline No & $13(6.4)$ & $100(49.5)$ & 1 & \\
\hline \multicolumn{5}{|c|}{ Refrigerator, no. (\%) } \\
\hline Yes & $3(1.49)$ & $38(18.81)$ & $0.526(0.050-0.628)^{*}$ & $0.072(0.007-0.745) *$ \\
\hline No & $21(10.40)$ & $140(69.31)$ & 1 & 1 \\
\hline \multicolumn{5}{|c|}{ Use of dustbin, no. (\%) } \\
\hline Yes & $4(2.0)$ & $21(10.4)$ & $1.584(0.491-5.105)$ & \\
\hline No & $19(9.40)$ & $158(78.2)$ & 1 & \\
\hline
\end{tabular}




\subsection{Prevalence of diarrhea and its covariates}

During last three months 144(71.3\%) suffered from any types of diseases. Among them, 22(10.9\%; 95\% CI: $6.685-15.3 \%$ ) children had suffered from diarrhea.

Bivariate analysis showed babies of literate mothers were less prone to have diarrhea than the illiterate mothers $(\mathrm{OR}=0.301$; 95\% CI: 0.128-0.829), Child from family economic income $\leq 10000$ BDT more likely to have diarrhea than monthly income $>10000$ BDT $(\mathrm{OR}=2.61$; 95\% CI: 1.024-6.973). Father's occupation $(\mathrm{OR}=0.326$; 95\% CI: $0.130-0.820)$, hand wash of babies before feeding ( $\mathrm{OR}=0.0156$; 95\% CI: 0.004-0.061), refrigerator (OR=0.526; 95\% CI: 0.050-0.628) were significantly associated with diarrhea. On the other hand, age of child, gender, family size, types of toilet, Trimming of Child's nail, some utensil use and dustbin had no significant association with diarrhea.

In the multivariate logistic regression analysis child of unemployed father's were more likely to have diarrhea than employed father (AOR=0.206; 95\% CI:0.070-0.610), babies who didn't use soap before feeding were more likely to have diarrhea as compared to those who used soap before feeding ( $\mathrm{AOR}=0.393 ; 95 \% \mathrm{CI}=0.102$ 0.511 ). Use of refrigerator was less likely to have diarrhea than never use it (AOR=0.072; 95\% CI: 0.007 0.745). However mother's education and family income had no significant association after adjusted for age and gender. The model was fit proved by Hosmer and Lemeshow goodness of fit test (Chi-square $=10.942$ and Pvalue $=0.205)$.

\section{Discussion}

Diarrhea recognized as a major cause of child morbidity and mortality in developing world (Dessalegn et al., 2011). This cross sectional study explored the association of hygienic practices with child diarrheal morbidity in a rural community. Maternal education, family income and child hand washing before feeding are the risk factors of diarrhea in this study area. Globally, diarrheal morbidity causes yearly 3.2 episodes among each under-five child (Kosek et al., 2003). Our study suggested eleven percent children suffered from diarrhea in three months recall. This prevalence is higher than overall rates of Bangladesh (BBS and UNICEF, 2016) and low income setting in Dhaka city (Chowdhury et al., 2015). Although rates were higher $(27.3 \%)$ in Ethiopia in two weeks recall (Hashi et al., 2016) and lower (9.3\%) in 24 hours recall in Kashmir, India (Ahmed et al., 2008). Vicinity to river might increase the rates in the study setting over national prevalence. Our study pointed that about half of respondent belongs to lower socio economic condition. Almost half of households earned less than $10000 \mathrm{BD}$ TK in the study area. Better economic condition of the family can easily improve hosing condition, convert unsanitary into sanitary and protect insect attack in kitchen.

Previous study has identified low maternal education as a risk factor for diarrhea (Kosek et al., 2008). We found an association with the maternal education with diarrheal episode in the study settings. Also, incidence was higher in low or no education in Sana'a, Yemen (Bin Mohanna and Al- Sonboli, 2018). Children whose mothers can't read and write were more likely to have diarrhea when compared with children whose mothers were literate (Gebru et al., 2014; Anteneh and Kumie, 2014). Education provides information regarding hygienic practices that might be prevents diarrhea (Shikur et al., 2013).

In Bangladesh, children of poor or middle class household face greater risk of illness (Kamal and Hasan, 2015; Ferdous et al., 2013). In this study, we found a higher risk of diarrhea in lower economic status. Economy is major constrains build up infrastructure that facilitate the hygienic practices of individuals as well as households. Moreover, poor settings accelerate transmission of disease pathogens results from ingestion of fecal contaminated water or food (Mandal et al., 2011). Another study in Khustia, Bangladesh diarrheal prevalence is quite alarming among children in lower socio economic condition (Khatun et al., 2013). Low income families suffer from diarrhoea because of inability to afford soap, aqua-guard and toilets (Rahman, 2006).

Occupation of the parents'/guardian might be cause of social-economic challenges in community (Karambu et al., 2013). We found an association with fathers' occupation with child diarrhea. This result is in accordance with Al- Mazrou et al. (1991). Similar findings also noted by Islam et al. (2015) in Bangladesh. Family income status in Bangladesh mainly depends on husband income (Khan et al., 2014). If the income is sufficient then obviously life style will be more improved and lower prevalence of diarrhea.

The disease is more prevalent among under-five children in the developing world due to lack of sanitation. Nearly $90 \%$ of diarrheal disease is attributed to inadequate sanitation (Bitew et al., 2017). In our study hand washing practices of mother and children were found considerably good. All mothers were washed their hand before and after feeding child, after self and child defecation which indicates good personal hygiene practices. But baby who do not use soap before feeding were 3.92 times more prone to diarrhea than who used soap. Natural habit touching everything of children causes cross contamination during feeding. Only water wash not proven enough to remove microorganism from babies hand. In case of cleaning utensil and cooking almost all 
household (95\%) use pond or canal water which might be harmful. Similar study done by Khatun et al. (2013) showed drinking water source whether safe or unsafe, has negligible impact on short term malnourishment of children Safe water source not only for drinking but also cleaning utensil and cooking is important to prevent chronic malnutrition and water born disease.

We found having refrigerator significantly reduce the diarrheal incidence in this area. Similar result was found in Barkina Faso (Bado et al., 2016). In contrast no change in prevalence between children of refrigerator owners and not having refrigerators after intervention (Luby et al., 2004). Refrigerator considered as live status indicator (Acharya et al., 2015) and essential parts of hygienic management and food.

The study was not free from limitations. In our study, cases were determined by participants themselves according to the definition of diseases, without biological or even practitioner's confirmation. Seasonal factor did not account into consideration. Child nutrition might be another cause excluded. The validity of the study may be limited by a cross-sectional rather than longitudinal design of the study and information contamination. However, this is the first study in the area and we believe, it raises awareness in the setting that will add valuable information to the existing healthcare service.

\section{Conclusions}

More than one in ten of child reported diarrhea in the study area. Family income and composition, fathers' occupation and child hand washing before feeding are the risk factors of diarrhea in this study area. Our study suggests there is significant effect of personal hygiene and kitchen hygiene practices on child diarrhea. Maintaining proper personal hygiene and improvement kitchen sanitation practices will lead to the reduction risk of diarrhea. Hence interventional program should be undertaken to reduce the risk of diarrhea in this setting.

\section{Conflict of interest}

None to declare.

\section{References}

Acharya D, V Khanal, JK Singh, M Adhikari and S Gautam, 2015. Impact of mass media on the utilization of antenatal care services among women of rural community in Nepal. BMC Res. Notes., 8: 345.

Khatun A, SS Rahman, H Rahman and H Sabir, 2013. A cross sectional study on prevalence of diarrhoeal disease and nutritional status among children under 5 years of age in Kushtia, Bangladesh. Science Journal of Public Health, 1: 56-61.

Ahmed SF, Farheen A, Muzaffar A and G Mattoo, 2008. Prevalence of diarrhoeal disease, its seasonal and age variation in under- fives in Kashmir, India. Int. J. Health Sci., 2: 126-133.

Alebel A, C Tesema, B Temesgen, A Gebrie, P Petrucka and GD Kibret, 2018. Prevalence and determinants of diarrhea among under-five children in Ethiopia: A systematic review and meta-analysis. PLoS ONE, 13: $\mathrm{e} 0199684$.

Al-Mazrou YY, KM Aziz and M Khalil, 1991. Association of parents' education and fathers' occupation with prevalence of diarrhoea among children less than five years of age in Saudi Arabia. J. Diarrhoeal Dis. Res., 9: 301-304.

Anteneh A and A Kumie, 2010. Assessment of the impact of latrine utilization on diarrheal diseases in the rural community of Hulet Ejju Enessie Woreda, East Gojjam Zone, Amhara Region. Ethiop. J. Health Dev., 24: 114

Anteneh ZA, K Andargie and M Tarekegn, 2017. Prevalence and determinants of acute diarrhea younger than five years old in Jabithenna district, Northwest Ethiopia, 2014. BMC Public Health, 17: 1.

Bado AR, AS Susuman and EI Nebie, 2016. Trends and risk factors for childhood diarrhea in sub-Saharan countries (1990-2013): assessing the neighborhood inequalities. Glob. Health Action, 9: 30166.

Balabanova D, A Mills, L Conteh, B Akkazieva, H Banteyerga, U Dash, L Gilson, A Harmer, A Ibraimova, Z Islam, A Kidanu, TP Koehlmoos, S Limwattananon, VR Muraleedharan, G Murzalieva, B Palafox, W Panichkriangkrai, W Patcharanarumol, L Penn-Kekana, T Powell-Jackson, V Tangcharoensathien and M McKee, 2013. Good Health at Low Cost 25 years on: lessons for the future of health systems strengthening. Lancet, 381: 2118-2133.

Bangladesh Bureau of Statistics (BBS), 2015. Population and Housing Census 2011. http://203.112.218.65:8008/WebTestApplication/userfiles/Image/PopCenZilz2011/Zila_Patuakhali.pdf.

Bangladesh Bureau of Statistics and UNICEF, 2016. Bangladesh Child Well-Being Survey 2016. Final Report. ISBN: 978-984-8969-26-7. 
Begum S, M Ahmed and B Sen, 2013. Impact of Water and Sanitation Interventions on Childhood Diarrhea: Evidence from Bangladesh. Available: http://www.eldis.org/document/A72180.

Bin Mohonna MA and N Al-Sonboli, 2018. Prevalence of diarrhea and related rsik factors among children aged under 5 in Sana'a, Yemen. Hamdan Med J., 11:29-33

Bitew BD, W Woldu and Z Gizaw, 2017. Childhood diarrheal morbidity and sanitation predictors in a nomadic community. Ital. J. Pediatr., 43: 91.

Chowdhury F, IA Khan, S Patel, AU Siddi, NC Saha, AI Khan, A Saha, A Cravioto, J Clemena, F Qadri and M Ali, 2015. Diarrheal Illness and healthcare seeking behavior among a population at high risk for diarrhea in Dhaka, Bangladesh. PLoS ONE, 10: e0130105.

Dessalegn M, A Kumie and W Tefera, 2003. Predictors of Under-Five Childhood Diarrhea: Mecha District, West Gojam, Ethiopia. Ethiop. J. Health Develop., 25: 192-200.

Ferdous F, SK Das, S Ahmed, FD Farzana, JR Latham and MJ Chisti, 2013. Severity of diarrhea and malnutrition among under five-year-old children in rural Bangladesh. Am. J. Trop. Med. Hyg., 89: 223-228.

Gebru T, M Taha and W Kassahun, 2014. Risk factors of diarrhoeal disease in under-five children among health extension model and non-model families in Sheko district rural community, Southwest Ethiopia: comparative cross-sectional study. BMC Public Health, 14: 395.

Getachew A, Guadu T, Tadie A, Gizaw Z, Gebrehiwot M, Cherkos DH, Menberu A and T Gebrecherkos, 2018. Diarrhea Prevalence and Sociodemographic Factors among Under-Five Children in Rural Areas of Norh Gondar Zone, Northwest Ethiopia. Int. J. Pediatr., ID 6031594, 8 page.

Gidudu J, D Sack, M Pina, M Hudson, K Kohl, P Bishop, A Chatterjee, E Chiappini, A Compingbutra, C da Costa, R Fernandopulle, Tk Fischer, P Haber, W Masana, MR de Menezes, G Kang, N Khuri-Bulos, LA Killion, C Nair, G Poerschke, B Rath, E Salazar-Lindo, R Setse, P Wenger, VC Wong and K Zaman, 2011. Diarrhea: case definition and guidelines for collection, analysis, and presentation of immunization safety data. Vaccine, 29: 1053 \pm 1071 .

Haque SA, 2006. Salinity Problems and Crop Production in coastal Regions of Bangladesh. Pak. J. Bot., 38: $1359-1365$.

Hashi A, A Kumie and J Gasana, 2016. Prevalence of diarrhoea and associated factors among under five children in Jigjiga District, Somali Region, Eastern Ethiopia. Open J. Prev. Med., 6: 233-246.

Islam MR, MK Hossain, MN Khan and MR Ali, 2015. Evidence of Socio-Demographic Effects on Child's Diarrhoea in Bangladesh. Journal of Health Science, 5): 1-5.

Kamal MM, MM Hasan and R Davey, 2015. Determinants of childhood morbidity in Bangladesh: evidence from the Demographic and Health Survey 2011. BMJ Open, 5: e007538.

Karambu S, V Matiru, M Kipto and J Oundo, 2013. Characterization and factors associated with diaeehoeal diseases caused by enteric bacterial pathogens among children aged five years and below attending Igembe Distric Hospital, Kenya. Pan Afr. Med. J., 16: 37.

Karambu S, V Matiru, M Kipto and J Oundo, 2013. Characterization and factors associated with diaeehoeal diseases caused by enteric bacterial pathogens among children aged five years and below attending Igembe Distric Hospital, Kenya. Pan Afr. Med. J., 16: 37.

Khan MN, MNI Mondal, N Hoque, MS Islam, and M Shahiduzzaman, 2014. A Study on quality of life of elderly population in Bangladesh. American Journal of Health Research, 2: 152-157.

Kosek M, C Bern and RL Guerrant, 2003. The global burden of diarrhoeal disease, as estimated from studies published between 1992 and 2000. Bull. World Health Organ., 81: 197-204.

Kosek M, PP Yori, WK Pan, MP Olortegui, RH Gilman, J Perez, CB Chavez, GM Sanchez, R Burga and E Hall, 2008. Epidemiology of highly endemic multiply antibiotic-resistant shigellosis in children in the Peruvian Amazon. Pediatrics, 122: e541-9.

Luby SP, M Agboatwalla, RM Hoekstra, MR Rahbar, W Billhimer, and BH Keswick, 2004. Delayed effectiveness of home-based interventions in reducing childhood diarrhea, Karachi, Pakistan. Am. J. Trop. Med. Hyg., 71: 420-427.

Mahapatra T, S Mahapatra, B Banerjee, U Mahapatra, S Samanta, D Pal, ND Chakraborty, B Manna, D Sur and S Kanungo, 2015. Predictors of Rational Management of Diarrhea in an Endemic Setting: Observation from India. PLoS ONE, 10: e0123479.

Mandal S, MD Mandal and NK Pal, 2011. Cholera: a great global concern. Asian Pac. J. Trop. Med., 4: 573580.

Mannan SR and MA Rahman, 2011. Exploring the link between food-hygiene practices and diarrhoea among the children of garments worker mothers in Dhaka. Anwer Khan Modern Medical College Journal, 1: 04-11. 
Mengistie B, Y Berhane and A Worku, 2013. Prevalence of diarrhea and associated risk factors among children under-five years of age in Eastern Ethiopia: a cross-sectional study. Open Journal of Preventive Medicine, 3: 446-453.

Osman FA, 2008. Health policy, programmes and system in Bangladesh achievements and challenges. South Asian Survey, 15: 263-288.

Rahman A, 2006. Assessing income-wise household environmental conditions and disease profile in urban areas: Study of an Indian city. Geo Journal, 65: 211-227.

Rahman AE, M Moinuddin, M Molla, A Worku, L Hurt, B Kirkwood, SB Mohan, S Mazumder, Z Bhutta, F Raza, S Mrema, H Masanja, D Kadobera, P Waiswa, R Bahl, M Zangenberg and L Muhe, 2014. Diarrhoeal deaths in seven low- and middle-income countries. Bull. World Health Organ. 92: 664-671.

Rony ZI, MSI Khan, MA Asgar, MR Begum and AG Kibria, 2016. Effects of saline water on health status of pregnant in coastal regions of Bangladesh. Asian J. Med. Biol. Res., 2: 55-61.

Shikur M, T Marelign and T Dessalegn, 2013. Morbidity and associated factors of diarrheal diseases among under five children in Arba-Minch district, Southern Ethiopia. Science Journal of Public Health, 1: 102-6.

Takanashi K, 2009. Survey of food - hygiene practices at home and childhood diarrhoea in Hanoi, Vietnam. J. Health Popul. Nutr., 27: 602-611.

Thiam S, N Aminata, F Samuel, S Mirko, Winkler, I Sy, A Jacques, Ndione, C Schindler, P Vounatsou J, O Aye and Cissé G, 2017. Prevalence of diarrhoea and risk factors among children under five years old in Mbour, Senegal: a cross-sectional study. Infect. Dis. Poverty., 6: 109.

Walker CL, MJ Aryee, C Boschi-Pinto and RE Black, 2012. Estimating diarrhea mortality among young children in low and middle income countries. PloS ONE., 7: e29151.

WHO, 2007. Water, Sanitation and Hygiene: Public Health and the Environment. Geneva. 